\section{Integrative Medicine \\ International}

\title{
Application of Laser Phoresis in Complicated Postmenopausal Osteoporosis
}

\author{
Elena Alexandrovna Belyaeva Alexander A. Khadartsev \\ Igor Alexandrovich Fedorischev Albert Sergeevich Sazonov
}

Medical Institute, Tula State University, Tula, Russia

\section{Key Words}

Osteoporosis · Analgesia · Osteoporotic fractures · Laser phoresis · Giasulf

\begin{abstract}
This study presents postmenopausal osteoporosis as a feature of metabolic systemic diseases of the skeleton, with abnormalities of the bone microarchitecture and reduction in bone mineral density, leading to an increased risk of fractures with a low level of trauma. The neurological manifestations of such fractures are uncharacteristic, representing a differential diagnostic indication. There is a change of posture in the form of hyperkyphosis of the thoracic spine and loss of height due to the increase in the number of modified vertebrae. The age of these patients is between 66 and 68 years, which limits the use of traditional analgesic preparations. The ionized gel 'Giasulf' was applied in 29 cases according to the developed technology. The description of the composition of the gel that provides maximum analgesic effect, reinforced by the influence of laser radiation on the cell membranes, is presented. A significant antinociceptive effect was obtained.

\section{Background}

Postmenopausal osteoporosis (OP) belongs to the metabolic systemic diseases of the skeleton, which are characterized by abnormalities of the bone microarchitecture and a decrease in bone mineral density, resulting in an increased risk of fractures with a low level of trauma. According to the epidemiological research, the early complication of postmenopausal OP is the fracture of the distal forearm. There is a short recovery period and, in most 
cases, the full restoration of functional abilities is achieved [1-4]. The fracture becomes a predictor of subsequent compressive fractures, with an increased probability during the postmenopausal period. This is due to the pathogenetic features of postmenopausal OP, namely early lesions in the trabecular bone, representing the body of the vertebrae. In individuals from the high-risk group aged $>55$ years (early and premature menopause, smoking, femur fractures on the mother's side, low bone mineral density, etc.), in the absence of the primary prevention, the clinical manifestation of $\mathrm{OP}$ is associated with compressive fractures of the vertebrae showing minimal trauma. The main clinical manifestation of compressive osteoporotic fractures is back pain of different degrees of intensity, having the tendency to chronicity. The neurological manifestations (symptoms of loss) are uncharacteristic for osteoporotic fractures, representing a differential diagnostic indication. Without a timely diagnosis and therapy, a change of posture in the form of hyperkyphosis of the thoracic spine and a decrease in height of more than $2 \mathrm{~cm}$ develops due to an increase in the number of modified vertebrae $[5,6]$.

Together with the specific therapy with biphosphonates, alphacalcidol, and denosumab, patients with fractures of the vertebrae need an adequate analgesic therapy. Most patients suffering from OP have comorbid diseases of the cardiovascular system, leading to destabilization with any additional pain symptom. However, in this group of patients, the selection of the analgesic therapy is complex. The median age of patients with osteoporotic fractures of the vertebrae ranges between 66 and 68 years, serving as a limitation for applying some pharmacological preparations, which are traditionally used for pain relief [nonsteroidal anti-inflammatory drugs (NSAIDs), simple analgesics]. It is known that in patients taking NSAIDs, fluid retention and increased blood pressure (BP) higher than 5-10 mm of mercury can be registered, which adversely affects their state, leading to arterial hypertension (AH) as well as chronic heart failure and increasing the risk of vascular disasters $[7,8]$. With the intake of NSAIDs, negative gastrointestinal reactions and a reduced excretory function of the kidneys more often develop in elderly patients. The analgesics, containing opiates (codeine), slow down the passage of the gastrointestinal tract, which results in the malabsorption of all other pharmaceuticals and the indiscriminate release of the acting substance from the preparations with a slow release. The additional risk factors of drug intolerance in patients $>60$ years of age are linked with the simultaneous application of pharmaceuticals and age-related changes of pharmacodynamics and pharmacokinetics. The pharmacodynamics and pharmacokinetics of pharmaceuticals are influenced by age-related factors, reducing the amount of fluid in an organism, the increase in adipose tissue, the decrease in the level of albumin in the blood serum, the decrease in the mass of the liver and the liver bloodstream, the impairment of oxidative metabolism, and the decrease in the concentration and excretory function of the kidneys.

The purpose of this study is to search for alternative methods of effective pain relief for acute and persistent pain syndrome in elderly patients with osteoporotic compressive fractures of the vertebrae.

\section{Materials and Methods}

Local therapy is one of the alternative methods, with the advantage of a direct influence on the invasion center, the decrease in the need in systematically prescribed medications and a good combination with the treatment, influencing the organism as a whole. With the purpose of analgesia, application therapy with ointment and gels and local treatment by means of physical factors and the combination of these methods are used $[9,10]$.

We used the treatment with electromagnetic waves emitted by a low-intensity laser. A laser irradiation causes the reconstruction of the biological processes associated with the 
regulation of the protection systems. In an organism, the biological reactions are activated not only in the irradiated tissues, but also in the regulatory centers. The biological effects of the laser therapy are displayed in the activation of the microcirculation in tissues and the stimulation of interstitial metabolism, the stimulation of the cellular growth and the reparation, the activation of protein synthesis and nucleic acids, the decrease in the intensity of oxidizing processes, and the restauration of the bioenergetic balance. The therapeutic effects of the laser therapy are many-sided and are displayed in the anti-inflammatory, antihydropic, thrombolytic, biostimulating, anesthetic, regenerative, and immunomodulatory action [11-13].

The advantages of semiconductor lasers, radiating in the near-infrared band of the electromagnetic spectrum $(0.87-0.91 \mu \mathrm{m})$, are a large depth of penetration of up to 6-7 $\mathrm{cm}$, a large beam divergence allowing to work without special defocusing optics, compactness, and the simplicity of service. The method of the projective transcutaneous irradiation was used for diseases of the musculoskeletal system. It was applied in a stable way and combined remote contact as well as remote scanning. The stable method consists in the installation of the nozzle in an immobile position on the irradiated zone for at least $1 \mathrm{~min}$. The contact method implies touching the surface of the body, thus the maximum energy efficiency on small sites is reached. In the remote sensing method, the radiator is at a distance of $1-3 \mathrm{~cm}$ from the irradiated surface, the defocusing of the beam being reached. The scanning way consists in the continuous movement of the radiator during the preset time. The course included 5-7 procedures [14].

In the laser phoresis method, a medicinal substance is applied to the site of the skin before the laser irradiation, which increases the therapeutic efficiency of the local application therapy due to the increase in tissue permeability of the applied remedy. In this way, the structural integrity of the drug is preserved. The additional ultrasound exposure can be performed directly before the laser therapy without interruption if necessary.

The fields of radiation on the lower thoracic and lumbar vertebrae are along the spine between the acantha and paravertebrally right and left, the method of irradiation used is stable contact and scanning, and the exposure time is 2-3 min (fig. 1).

In this research, the 'Giasulf' gel was chosen as a preparation for the laser phoresis, the basic acting substance of this gel is hyaluronic acid. Hyaluronic acid is a nonsulphated glycosaminoglycan; in the human organism, it is found overall and is one of the basic components of the intercellular substance. In recent years, it has found a wide application in medicine for the treatment of skin affections, the pathology of the eye, diseases and injuries of the joints, as the inducer of osteogenesis, the regulator of phagocytic function, and the means of controlled delivery of drugs. Hyaluronic acid plays the most important role in the support of the normal structure and the function of cartilaginous structures. Along with the collagen, it is the main component of the basic substance of cartilage, enhances the synthesis of proteoglycans and blocks metalloproteinases destroying a cartilage [9].

The second basic component contained in the gel is dimethyl sulfoxide (Dimexidum), providing an anti-inflammatory, analgesic, and fibrinolytic action and being 'a conductor' for associated medicinal components. Additional components, such as lidocaine, herbal extracts (of horse chestnut, aralia, St. John's wort, bird cherry, wormwood), nipacet, and Carbomer, are used for the attainment of the maximum local anesthetic, anti-inflammatory, and antihydropic effects [13]. Feature of the use of this gel in our study was preliminary ionization by means of a specially designed apparatus (fig. 2).

The ALSTAT apparatus has a dual purpose: (1) it functions as the ionizer of liquids, wateralcohol solutions, and gels. (2) It contributes to the implementation of the transdermal ion phoresis. 
Belyaeva et al:: Application of Laser Phoresis in Complicated Postmenopausal Osteoporosis

Fig. 1. Zones of laser phoresis: lower thoracic (1-3) and lumbar vertebrae $(4,5)$.
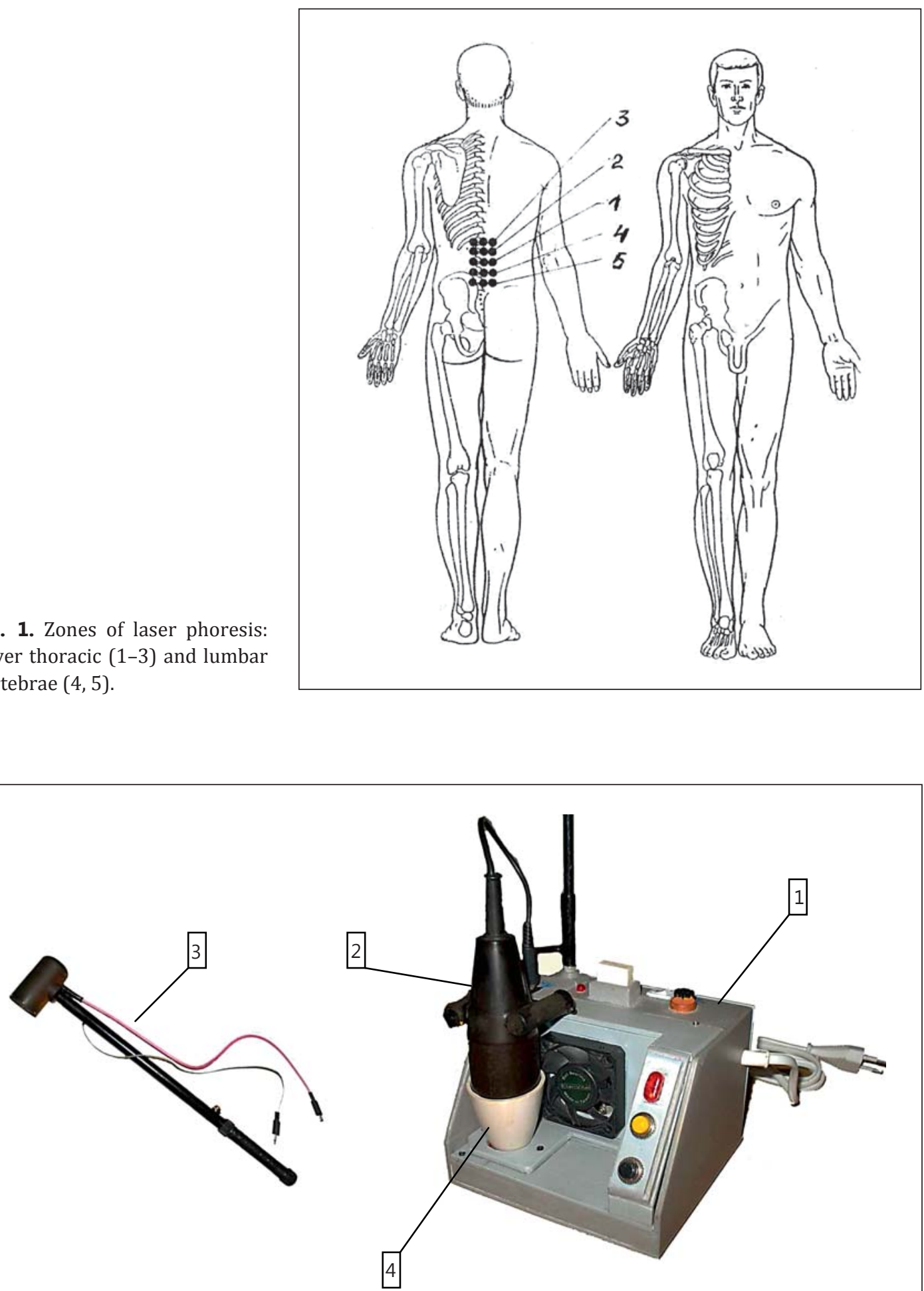

Fig. 2. The ALSTAT apparatus is presented: $1=$ high voltage unit; $2=$ negative ion generator; $3=$ external ionizer, and $4=$ container for an ionisable substance. 
Belyaeva et al.: Application of Laser Phoresis in Complicated Postmenopausal Osteoporosis

An ionisable substance is placed in a container with metal bottom, and a block of the corona needles is placed coaxially with the container at a distance of $1-2 \mathrm{~cm}$. When the voltage on the needles is $25-30 \mathrm{~kW}$, negative lightions flow from the corona needles and are embedded under the action of a strong electric field in an ionisable substance, giving it a negative charge and changing the properties of the substance. After ionization, the substance is injected into the body by any method available.

In the case of transdermal ion phoresis, a medicinal substance in the form of ointments, gels, or moistened wipes is applied to the skin. The ionizer is placed on the rod of insulating material, which has the ability to remotely scan a medicinal substance. A passive electrode of the ionizer has electrical contact with the patient, but in such a way that the lines of the ion current are not passed through the area of the heart. The light ions, flowing down from the corona needles of the ionizer bombard the medicinal substance, produce ionization of molecules, which in turn penetrate through the skin into the body.

The medical laser phoresis was carried out in a group of 29 patients aged between 58 and 82 years with the pain syndrome due to compressive fractures of lower thoracic and lumbar vertebrae. Selection criteria were radiographically verified fractures of the thoracic and lumbar vertebrae, associated with OP and accompanied by a pain syndrome of different duration and degree of manifestation. Criteria for exclusion were cancer diseases in the anamnesis, skin diseases, and age-related mental disorders.

For the specific OP therapy, all patients took drugs from the group of biphosphonates. Estimation of the pain syndrome according to the visual analogue scale (VAS) was made before the treatment and after the end of the full course of 7 procedures. For the estimation of tolerability, a poll and a clinical research with the measurement of BP after each procedure and the determination of average daily values of BP before the treatment by laser phoresis and after its completion were conducted.

The Ethics Committee of the Medical Institute, Tula State University, approved the protocol of the present survey.

\section{Results}

At treatment start, the main complaint of the patients was back pain. Pain intensity varied from 67 to $89 \mathrm{~mm}$ according to the VAS. A total of 27 patients completed the treatment. The regress of the pain syndrome was noted for all patients by the end of the full course. On average, a decrease in pain intensity of up to $68 \%$ from the reference value has been registered.

The pain VAS is a unidimensional measure of pain intensity. It is the most simple, convenient, and widely used measure in everyday practice [15-19]. The VAS is a straight line of fixed length of usually $10 \mathrm{~cm}$ or $100 \mathrm{~mm}$ (both measures are used). The patient is asked to place a line perpendicular to the VAS line at the point that represents their pain intensity. The following cut points exist on the pain VAS: the initial point - no pain $=0$, then mild pain, moderate pain, strong pain, ultimate pain, and unbearable pain -10 .

There are several indications that facilitate the understanding of the result: the first group of indications is: $0=$ the pain does not worry the patient; $2=$ the pain is insignificant; 4 = the pain is a little worrying; 6 = the pain is significantly worrying; $8=$ the pain is significant, the patient's consciousness is focused on the pain, and $10=$ the pain is so severe that the patient submits to it; he cannot suffer this pain. The second group of indications is: excellent (no pain), good (30 $\mathrm{mm}$ ), satisfactory $(60 \mathrm{~mm})$, and unsatisfactory (over $60 \mathrm{~mm}$ ). The evaluation of the effect of the laser phoresis was carried out on indication 2 [5].

The tolerability was estimated by a doctor and a patient separately. A local allergic reaction was noticed in two cases and appeared as dermahemia and the occurrence of itching 
Table 1. Estimation of tolerability of laser phoresis by a doctor and a patient

\begin{tabular}{l|l}
\hline Integr Med Int 2016;3:17-23 \\
\hline DOI: 10.1159/000442669 & $\begin{array}{l}\text { C } 2016 \text { The Author(s). Published by S. Karger AG, Basel } \\
\text { www.karger.com/imi }\end{array}$ \\
\hline
\end{tabular}

Belyaeva et al.: Application of Laser Phoresis in Complicated Postmenopausal Osteoporosis

\begin{tabular}{lll}
\hline Tolerability & $\begin{array}{l}\text { Patient's estimation } \\
(\mathrm{n}=29)\end{array}$ & $\begin{array}{l}\text { Doctor's estimation } \\
(\mathrm{n}=29)\end{array}$ \\
\hline Excellent & 21 & 21 \\
Good & 2 & 4 \\
Satisfactory & 4 & 2 \\
Unsatisfactory & 2 & 2 \\
\hline
\end{tabular}

after the application of the gel. In these cases, the therapy was stopped. The estimation of the therapy by a doctor and a patient is shown in table 1 .

An unsatisfactory estimation was associated with the occurrence of an individual reaction of the intolerance to the components of the drug and the cessation of the therapy. A satisfactory tolerability in a doctor's opinion was associated with the transitory decrease in BP after the first laser phoresis procedures in patients with atherosclerotic disease and a cerebrovascular pathology.

The change of BP during treatment and at the end of the course of therapy is of interest. No patient had an increase in BP after the laser phoresis procedure, and at the end of the treatment course, the decrease in the average systolic BP was up to $5.8 \mathrm{~mm}$ of mercury, which can be considered as a protective factor in the case of a cardiovascular pathology.

One of the main aims of the use of laser phoresis with the Giasulf gel was the decrease in doses of analgesics and the treatment of elderly patients who refuse systematic analgesic therapy, having a wide range of contraindications and side reactions and a low profile of compatibility with hypotensive and antianginal medicines in patients with $\mathrm{AH}$ and coronary heart disease. A total of $27 \%$ of patients could completely avoid the use of systematic analgesics, $62 \%$ of patients decreased the daily dose of analgesic drugs more than twice, and $11 \%$ returned to the usual doses of analgesics.

Another important assessment criterion of applying the laser phoresis method was the increase in the patients' motor activity, which was decreased because of the intensity of the pain syndrome. Motor activity is of great significance for elderly patients as it contributes to maintaining coordination, the prevention of physical weakness and is a protective factor in relation to the bone tissue, preventing the rapid progression of OP on the background of hypokinesia. During physical activity, the capillary blood flow improves not only in skeletal muscles, but also in all organs and tissues, including the brain and heart, which is especially important for patients with comorbid diseases of the cardiovascular system, i.e. AH and coronary heart diseases.

The estimation of the expansion of the motor activity was conducted by the survey questionnaire method, containing 12 questions about daily household activities. According to the poll results, the expansion of the motor activity has been marked in 17 patients after the end of the course of laser phoresis and is associated with the decrease in pain at rest and at motion.

\section{Discussion}

Thus, the laser phoresis method with the use of the Giasulf gel is safe for elderly patients with concomitant multiorgan pathology with osteoporotic compressive fractures of vertebrae, and it can be applied as a rehabilitation method of treatment for the decrease in the doses of systematic analgesics and the expansion of motor activity. In addition, it has a 
Belyaeva et al.: Application of Laser Phoresis in Complicated Postmenopausal Osteoporosis

possible influence on further prognosis, taking into account the additional positive systematic effects.

The technology was defined according to the research results of recovery treatment of postmenopausal OP: (1) the basic purpose of the application of ointments and gels with NSAIDs - twice daily (morning and evening) on the spine. (2) Ionization by the Giasulf gel within 5 min. (3) The use of the ionized gel on the spine. (4) The impact of low-energy laser radiation in the infrared range, the wavelength of $0.87-0.91 \mu \mathrm{m}$ for 3-4 min by the stable method, then another 4-5 min by the scan method. (5) The treatment course consists of 7-10 procedures.

\section{Disclosure Statement}

The authors declare that there are no conflicts of interest regarding the publication of this article.

\section{References}

1 Belyaeva EA: Methodological approaches to the prevention of osteoporosis at the regional level. Med Sci 2009; 1:20-22.

2 Belyaeva EA: X-ray diagnosis and prevention of fractures in patients with steroid osteoporosis. Bull New Med Technol 2010;1:43-44.

3 Belyaeva EA: Epidemiological characteristics of patients with primary postmenopausal osteoporosis. Bull New Med Technol 2011;4:198-201.

4 Belyaeva EA, Khadartsev AA: A systematic approach to medical rehabilitation of patients with osteoporosis. Syst Anal Control Biomed Syst 2010;1:13-17.

5 Belyaeva EA, Khadartsev AA: Recovery Therapy of the Complicated Postmenopausal Osteoporosis. Tula, PH «Grif and K», 2010.

6 Shabalin VN (ed): Manual on Gerontology. Moscow, Zitadel-treid, 2005.

7 Belyaeva EA: Actual issues of recovery therapy in degenerative diseases of the skeleton and comorbid pathology. Bull New Med Technol 2011;1:28-31.

8 Grigoriev AI, Khadartsev AA, Fudin NA, et al: Electro Laser Myostimulation and Laser Phoresis of Biologically Active Substances in Sport: Methodological Guide. Tula, RIF «Infra», 2005.

9 Avdeeva OS, Belyaeva EA, Khadartsev AA, Fedorischev IA: Experience in the use of local therapy application hyaluronate containing gel 'Giasulf' at the articular syndrome. Bull New Med Technol 2008;4:183-185.

10 Belyaeva EA, Khadartsev AA: Theoretical aspects of restorative treatment of osteoporosis with comorbid pathology. Bull New Med Technol 2010;3:96-98.

11 Belyaeva EA: Therapy of osteoporosis in early postmenopausal period. Pharmateka 2008;12:42-46.

12 Belyaeva EA: Therapy of postmenopausal osteoporosis. Con Med 2008;2:40-43.

13 Belyaeva EA, Fedorischev IA: Laser phoresis of the hyaluronate containing gel 'Giasulf' at the complicated postmenopausal osteoporosis. Bull New Med Technol 2010;1:36-38.

14 Belyaeva EA, Kupeev VG, Khadartsev AA: New technology of safe analgesic therapy at the complicated osteoporosis. Bull New Med Technol 2010;3:122-124.

15 Huskisson E: Visual analogue scales; in Melzack R (ed): Pain Measurement and Assessment. New York, Raven Press, 1983, pp 33-37.

16 Weaver ME, Lowe NK: A critical review of visual analogue scales in the measurement of clinical phenomena. Res Nurs Health 1990;13:227-236.

17 Revill SI, Robinson JO, Rosen M, Hogg MI: The reliability of a linear analogue for evaluating pain. Anaesthesia 1976;31:1191-1198.

18 Seymour RA: The use of pain scales in assessing the efficacy of analgesics in post-operative dental pain. Eur J Clin Pharmacol 1982;23:441-444.

19 Price DD, McGrath P, Raffi A, Buckingham B: The validation of visual analogue scales as ratio scale measures for chronic and experimental pain. Pain 1983;17:45-56. 